\title{
Cost-effectiveness analysis of alirocumab in high cardiovascular-risk patients in Italy
}

\author{
Massimiliano Povero ${ }^{1}$, Lorenzo Pradelli ${ }^{1}$, Andrea Serra ${ }^{2}$, Francesca Fanelli ${ }^{2}$, \\ Luca Gazzi ${ }^{2}$ \\ AdRes HE\&OR, Turin, Italy \\ Sanofi S.p.A., Milan, Italy
}

\begin{abstract}
OBJECTIVE: Dyslipidemia, in particular elevated total and low-density lipoprotein cholesterol (LDL-C), results in atherosclerosis and increases the risk of cardiovascular (CV) events. Despite treatment with statins, many patients fail to reduce their LDL-C enough to optimally minimize their risk. Novel therapy alirocumab, on top of background statin therapy, resulted efficacious in lowering CV risk by reducing LDL-C levels. Aim of the present paper is to evaluate the cost-effectiveness of alirocumab in high cardiovascular-risk patients in Italy

METHODS: A 1-year cycles Markov model was developed to evaluate the cost-effectiveness of statins at maximum dose tolerated plus ezetimibe (MDTS+E) with or without alirocumab. Target population consisted of patients with high baseline risk of CV events. Patients entered the model in stable disease and could experience a non- fatal CV event (acute coronary syndrome, elective revascularization or ischemic stroke) or die. Results from the ODYSSEY trial were used to evaluate CV risk reduction due to alirocumab add-on. Pharmaceutical, CV events, and LDL-C levels' detection costs are considered in the analysis from the perspective of Italian National Health Service.

RESULTS: Simulated cohort was 75 years old on average, $66 \%$ male, $42 \%$ diabetes mellitus and baseline LDL-C level equal to $121 \mathrm{mg} / \mathrm{dl}$. Furthermore, $96 \%$ of subjects were hospitalized in the last 12 months. Alirocumab used as an add-on to MDTS+E was more costly ( $€ 45,358$ vs $€ 13,208$ ) but more effective (8.01LY vs 6.33LY) than MDTS+E, leading to an incremental cost effectiveness ratio of $€ 19,158$ per LY. At a willingness to pay threshold of $€ 30,000$ per LY, alirocumab had $96 \%$ probability to be cost effective vs. MDTS+E alone. Results were relatively more favorable in the patient subset with recent $\mathrm{CV}$ event ( $<12$ months from index).

CONCLUSION: The results indicate that alirocumab in addition to MDTS+E is cost-effective versus MDTS+E alone in a representative cohort of high CV risk patients in Italy.
\end{abstract}

\section{Keywords}

Dyslipidemia; Cardiovascular events; Statin therapy; Alirocumab; Cost-effectiveness

\section{INTRODUCTION}

The pathophysiology of atherosclerotic cardiovascular disease (ASCVD) is related to multiple agents such as abnormal blood lipids levels, high systolic blood pressure, diabetes, tobacco smoking and abdominal obesity [1].

Lipids, in particular low-density lipoprotein (LDL) and other apolipoprotein B (apoB)containing lipoproteins with small diameter, can go through the arterial intima and induce the atherosclerotic plaque formation and are considered important factors related to atherosclerotic disease. Moreover, the probability of cholesterol intimal retention and the progressive development of atherosclerotic lesions increase with the LDL blood levels in a dose dependent manner [2].

LDL pathways and its effects have been deeply studied in the past 100 years highlighting the function of different molecular structures like the LDL-receptors (LDL-R), HMG-CoA reductase, Sterol Regulatory Element-binding Protein (SREBP) and proprotein convertase subtilisin-like/kexin type 9 (PCSK9). The knowledge of these pathways allowed to develop

Corresponding author Massimiliano Povero m.povero@adreshe.com 
several LDL lowering drugs able to interact with molecular mechanisms and decrease the risk of cardiovascular (CV) events [3].

One of the molecules discovered most recently is PCSK9, a protease that binds the LDL$\mathrm{R}$ and promotes their degradation, thus reducing the clearance of LDL plasma levels [3]. Alirocumab is a human monoclonal antibody that binds to PCSK9 with high affinity and specificity, inhibiting the interaction between this protease and LDL-R. This phenomenon increases the number of LDL-R available to clear LDL, thereby lowering LDL cholesterol (LDL-C) levels [4].

Efficacy of alirocumab was evaluated on different populations with inadequate response to lipid-lowering therapy. The ODYSSEY OUTCOMES trial [5] compared alirocumab with placebo, added to high-intensity or maximum-tolerated statin treatment, as secondary prevention after acute coronary syndrome (ACS). This multicenter, randomized, double-blind, placebocontrolled trial involved almost 19,000 patients and showed a reduction of the risk of recurrent ischemic cardiovascular events among those who received alirocumab compared to those who received placebo (hazard ratio [HR]: 0.85; 95\% confidence interval [CI]: 0.78-0.96). A post hoc analysis showed the reduction was more pronounced in patients with baseline LDLC greater than $100 \mathrm{mg} / \mathrm{dl}$ (HR: 0.76, 95\% CI: 0.65-0.87).

Aim of the present paper is to evaluate both clinical and economic consequences due to alirocumab in addition to a "background therapy" defined as the maximum tolerated dose of high intensity statins and ezetimibe (MDTS+E) in patients with high baseline risk of CV events: patients with acute coronary syndrome, myocardial infarction or unstable angina. The analysis adopted the Italian National Health Service (NHS) perspective, hence only direct medical cost was considered in the present analysis [6]; according to international literature referring to the Italian context, a discount rate equal to $3.5 \%$ has been applied for both costs and benefits [7].

\section{METHODS}

The cost-effectiveness analysis (CEA) was based on a 1-year cycles cohort Markov model, developed through MS Excel ${ }^{\circledR}$, with a lifetime perspective (time horizon of 40 years). The model allows to evaluate clinical and economic consequences of alirocumab, simulating the evolution of a hypothetical population with or without the addition of MDTS+E. The risk of $\mathrm{CV}$ events is estimated at the begin of the simulation considering population baseline characteristics - age, sex, risk factors and LDL plasma levels - and changes over time depending on therapies received. Costs include drugs and

\begin{tabular}{|c|c|c|}
\hline & ACS 0-12 & ACS $>12$ \\
\hline Mean age ${ }^{1}$ (years) & & \\
\hline$<55$ & & \\
\hline$<65$ & & \\
\hline$<75$ & & \\
\hline$<80$ & & \\
\hline Sex (male, \%) & & \\
\hline Comorbidities (DM, \%) & & \\
\hline Mean LDL-C level' ${ }^{2}(\mathrm{mg} / \mathrm{dl})$ & 121.06 & 126.02 \\
\hline$<130$ & $73 \%$ & $64.9 \%$ \\
\hline $130-160$ & $21.9 \%$ & $24.3 \%$ \\
\hline$>160$ & $5.1 \%$ & $10.8 \%$ \\
\hline
\end{tabular}
$\mathrm{CV}$ events management.

\section{Target population}

Simulated cohort consists of patients characterized by high risk of CV events, specifically patients with ACS, myocardial infarction or unstable angina who experienced at least one hospitalization - within the last 12 months (ACS 0-12) or previous to the last 12 months (ACS $>12$ ).

Baseline characteristics (age, sex, concomitant diabetes mellitus, and mean LDL-C levels) are estimated according to a primary care database regarding more than 80,000 patients treated with statins in Italy (Table I) [8].

According to the AIFA (Italian medicines agency) reimbursement agreement, the minimum LDL-C level considered to start the therapy is fixed at $100 \mathrm{mg} / \mathrm{dl}$ for secondary prevention therefore only patients with baseline LDL-C level >100 mg/dl were considered in the analysis.

Baseline risks regarding $\mathrm{CV}$ events are not available specifically for our population, hence data derived from UK-THIN database and used in the alirocumab CEA submission 


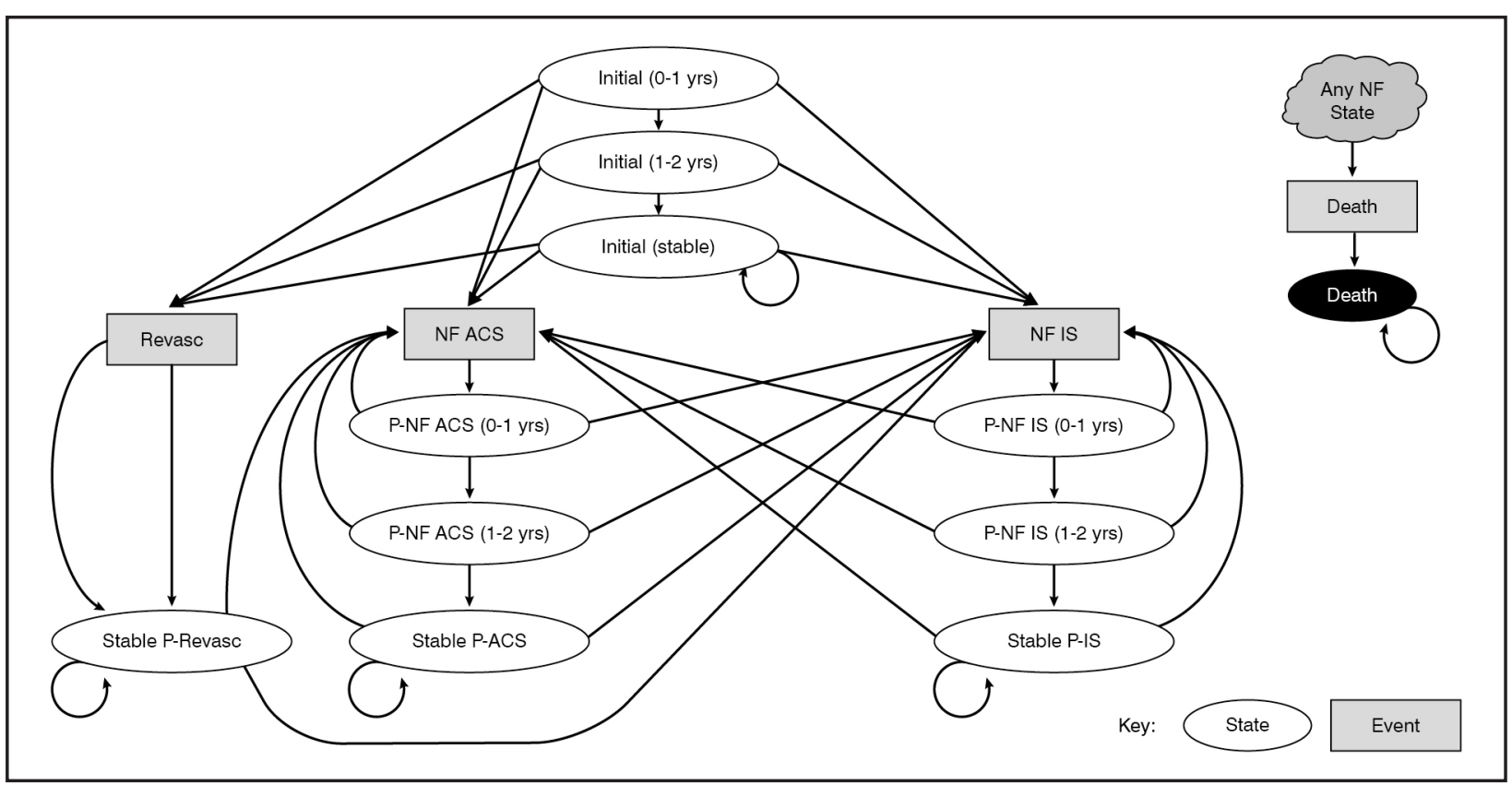

Figure 1. Markov model

ACS = acute coronary syndrome; IS = ischemic stroke; MI = myocardial infarction, NF = non-fatal; P- = post; Revasc = elective revascularization

to NICE were considered [9], and adjusted for the national population: risk of CV events, divided by kind of event, is specific for population age and basal LDL-C level (Table II). Moreover, as diabetes mellitus is a risk factor for $\mathrm{CV}$ events, data specific for patients with and without diabetes were reported.

\section{Model: description and risk}

Patients have 2 possible initial states based on previous hospitalization for CV events: patients ACS 0-12 months start in state "Initial (0-1 yr)", then the following year move to "Initial ACS 1-2 yrs" and only at the beginning of the third year are considered as "Stable". Patients in the group ACS >12, start from "Initial ACS (1-2 yrs)" and move to the "Stable" state the following year. From the "Stable" state, patients can experience a non- fatal CV event (acute coronary syndrome, elective revascularization or ischemic stroke) according to specific probabilities, or death. (Figure 1).

Based on expert opinion and to simplify the model, non-fatal myocardial infarction and unstable angina are considered as a unique event which is calculated as a weighted mean of both risks. Elective revascularization is included in the model as it can modify future risk of $\mathrm{CV}$ events; however, this state is assumed to be only prior to ACS or ischemic stroke

\begin{tabular}{lcc|cc}
\hline \multirow{2}{*}{ Population } & \multicolumn{2}{c}{ With DM } & \multicolumn{2}{c}{ Without DM } \\
\cline { 2 - 5 } & ACS 0-12 & ACS >12 & ACS 0-12 & ACS > 12 \\
\hline CV annual risk (\%) & 24.80 & 9.40 & 15.60 & 7.20 \\
- MI NF & 5.20 & 1.70 & 4.10 & 1.50 \\
• Unstable angina & 3.30 & 1.20 & 2.40 & 0.70 \\
- ACS & 8.50 & 2.80 & 6.50 & 2.30 \\
• RVSC & 4.90 & 0.90 & 3.60 & 0.70 \\
- IS NF & 1.70 & 1.00 & 0.80 & 0.70 \\
- All-cause death & 9.60 & 4.70 & 4.70 & 3.60 \\
Mean UK-THIN LDL-C (mg/dl) & 88.9 & 90.5 & 100.5 & 96.7 \\
Mean UK-THIN age (years) & 70.9 & 72 & 69.5 & 72.1 \\
\hline
\end{tabular}

Table II. Baseline risk for CV events

ACS = acute coronary syndrome (non-fatal MI and unstable angina); ACS 0-12 = patients with acute coronary syndrome, myocardial infarction or unstable angina with hospitalization within the last 12 months; ACS >12 = patients with acute coronary syndrome, myocardial infarction or unstable angina with hospitalization previous to the last 12 months; CV = cardiovascular; DM = diabetes mellitus; IS = ischemic stroke; LDL-C = low-density lipoprotein cholesterol; MI = myocardial infarction; NF = non-fatal; RVSC = revascularization 


\begin{tabular}{lc}
\hline \multicolumn{1}{c}{ Event } & HR (95\% Cl) \\
\hline Non-fatal myocardial infarction or unstable angina & $0.74(0.70-0.77)$ \\
Revascularization & $0.76(0.72-0.78)$ \\
Ischemic stroke & $0.79(0.74-0.85)$ \\
All-cause death & $0.91(0.88-0.93)$ \\
\hline
\end{tabular}

Table III. Hazard ratio reduction in baseline $\mathrm{CV}$ risk related to $1 \mathrm{mmol} / \mathrm{I}$ reduction of $L D L-C$

$\mathrm{Cl}=$ confidence interval; $\mathrm{CV}=$ cardiovascular; $\mathrm{HR}=$ hazard ratio; LDL-C = low-density lipoprotein cholesterol

\begin{tabular}{lcc}
\hline \multirow{1}{*}{ Event } & \multicolumn{2}{c}{ HR (95\% CI) } \\
\cline { 2 - 3 } & \multicolumn{1}{c}{ Year 1 } & Years 2+ \\
\hline Non-fatal myocardial infarction & $0.95(0.74-1.23)$ & $0.63(0.48-0.82)$ \\
Unstable Angina & $0.68(0.24-1.91)$ & $0.36(0.11-1.12)$ \\
Revascularization & $0.89(0.70-1.14)$ & $0.66(0.51-0.86)$ \\
Ischemic stroke & $0.43(0.21-0.91)$ & $0.75(0.44-1.30)$ \\
All-cause death & $0.79(0.51-1.22)$ & $0.67(0.50-0.89)$ \\
\hline
\end{tabular}

Table IV. Hazard ratios of $\mathrm{CV}$ risk related to treatment from ODYSSEY OUTCOMES trial

$\mathrm{Cl}=$ confidence interval; $\mathrm{CV}=$ cardiovascular; $\mathrm{HR}=$ hazard ratio
(IS) as both these events precede elective revascularization. In case of ACS or IS event, two subsequent states are planned in order to model the clinical course till a new stable state is achieved post ACS or IS (assumed to be two years after the event).

Baseline risk of $\mathrm{CV}$ events, derived from UK-THIN data (Table II), are adjusted for LDL-C levels and age of Italian population by applying:

- HR estimated in the CTT study [10], a meta-analysis of 27 randomized clinical trials including about 170,000 patients (Table III); each HR measures the reduction in risk of $\mathrm{CV}$ event related to a reduction of $1 \mathrm{mmol} / 1$ of LDL-C;

- Relative risks (RR) for each year increment with respect to the UK-THIN baseline age every year; RR is equal to 1.03 in case of non-fatal event and 1.10 for fatal events, according to Wilson et al. [11].

Moreover, to take into account the effect of previous $\mathrm{CV}$ events, risk of death related to $\mathrm{CV}$ event is further increased with a RR equal to 1.5 according to Smolina et al. [12].

The change over time of CV event risk due to alirocumab is simulated directly from ODYSSEY OUTCOMES trial [5] results (Table IV). Data are broken down between first and subsequent years, aiming to include the delayed effect of the treatment observed in KaplanMeier curves of trial (curves separate only after few months after the beginning of treatment).

\section{Costs}

According to the Italian NHS perspective, only direct health costs have been considered. Specifically costs of drugs (alirocumab, statins and ezetimibe), costs related to CV events management, and costs related to LDL-C levels' detection.

Acquisition cost of alirocumab is estimated considering a pen delivery system prefilled with $75 \mathrm{mg}$ of active substance, administered subcutaneously every two weeks [4,13]. We assumed 100\% compliance and lifelong treatment. Annual treatment cost is estimated considering the ex-factory prices at net of VAT, applying the mandatory discount $(5 \%+5 \%$ reduction $)$ and price reductions negotiated among the pharmaceutical company, Local Health Units and Hospital Units .

The analysis regarding statins consumption in Italy [8], highlights that $74 \%$ of patients take atorvastatin and $26 \%$ take rosuvastatin. The mean annual cost of treatment, considering for each statin the reference price in Italian NHS [14], is equal to $€ 149.86$, while mean annual cost treatment with ezetimibe is equal to $€ 293.83$ assuming $100 \%$ adherence for all the analysis.

$\mathrm{CV}$ event costs are broken down into costs related to the event and the incremental follow-up costs (rehabilitation, adjunctive therapies, complications, etc.). Events considered and related costs, divided into event related cost (ERC) and follow-up cost (FC) are: non-fatal myocardial infarction [15] $(\mathrm{ERC}=€ 18,457 ; \mathrm{FC}=€ 3,206)$, unstable angina [15] (ERC $=€$ 3,069 ; no FC), revascularization by coronary artery bypass grafting (CABG) or percutaneous coronary interventions $(\mathrm{PCI})[16](\mathrm{ERC}=€ 7,403$; no $\mathrm{FC})$, ischemic stroke [15] (ERC $=€$ $8,794 ; \mathrm{FC}=€ 10,675)$, death due to cardiovascular causes [16] (ERC $=€ 4,018$, no FC). All data are up to date per 2019 using the Italian inflation rate for healthcare sector [17].

The cost for LDL-C level detection, needed for assessment of the eligibility to alirocumab, is calculated considering the cost for a complete lipid profile (HDL-C, LDL-C, total, and triglycerides) using Italian National tariffs [18].

\section{Sensitivity analysis}

A probabilistic sensitivity analysis (PSA) was conducted to take into account the input parameter uncertainty and its global influence on final results. PSA included 1,000 simulations, in each iteration input parameters are sampled from ad-hoc distributions centered on the base 


\begin{tabular}{lccc}
\hline & Alirocumab+MDTS+E & MDTS+E & Delta \\
\hline LY & 8.01 & 6.33 & 1.68 \\
Total cost $(\boldsymbol{\epsilon})$ & $45,358.38$ & $13,208.46$ & $32,149.91$ \\
Drug cost & $37,099.50$ & $2,807.64$ & $34,291.87$ \\
CV events & $8,245.95$ & $10,400.83$ & $-2,154.88$ \\
- ACS & $4,261.79$ & $6,048.46$ & $-1,786.67$ \\
• Revascularization & 727.71 & 793.49 & -65.78 \\
• Non-Fatal IS & $1,429.43$ & $1,582.08$ & -152.65 \\
• CV deaths & $1,827.02$ & $1,976.80$ & -149.78 \\
LDL-C detection & 12.93 & 0.00 & 12.93 \\
ICER $(\boldsymbol{\epsilon})$ & & & $19,158.12$ \\
\hline
\end{tabular}

Table V. Cost-effectiveness analysis: base case results

ACS = acute coronary syndrome; CV = cardiovascular; $\mathrm{E}=$ ezetimibe; ICER = incremental cost effectiveness ratio; IS = ischemic stroke; LDL-C = lowdensity lipoprotein cholesterol; MDTS = maximum dose tolerated statins

case value (i.e. the mean of each distribution corresponds to the value used in the base case analysis). In particular, baseline cohort characteristics ( $\%$ of male, mean age, $\%$ of diabetic patients, CV event risks), alirocumab efficacy and mean cost per event, have been sampled from normal distributions; HRs, RRs and basal LDL-C levels from log-normal distributions. If not available, standard error has been approximated as the range divided by 6 [19].

\section{RESULTS}

Patients with a recent acute coronary syndrome (ACS 0-12 population) represent almost $96 \%$ of the total cohort. The addition of alirocumab to MDTS+E gives an expected improvement in overall survival of 1.68 life-years (LY), this gain requires an increase in lifetime costs equal to $€ 32,171$. The economic impact is mainly due to cost of therapy $(€+34,292)$ and partly compensated by the reduction in $\mathrm{CV}$ events rate producing a saving of $€-2,155$. Incremental cost effectiveness ratio (ICER) results in $€ 19,158$ per LY (Table V). Results were relatively more favorable in the patient subset with $\mathrm{CV}$ event $<12$ months from index (Table IA and Table IIA in Appendix).

Results of 1,000 simulations carried out for PSA (Figure 2), considering the uncertainty regarding input parameters, highlight a limited variability of estimated ICER $(95 \% \mathrm{CI}$ : $€$ 11,609-31,475 per LY).

The cost-effectiveness acceptability curve shows that at the maximum threshold of $€ 30,000$ per LY [20], the probability that alirocumab is cost-effective was equal to $96 \%$ (Figure 3).

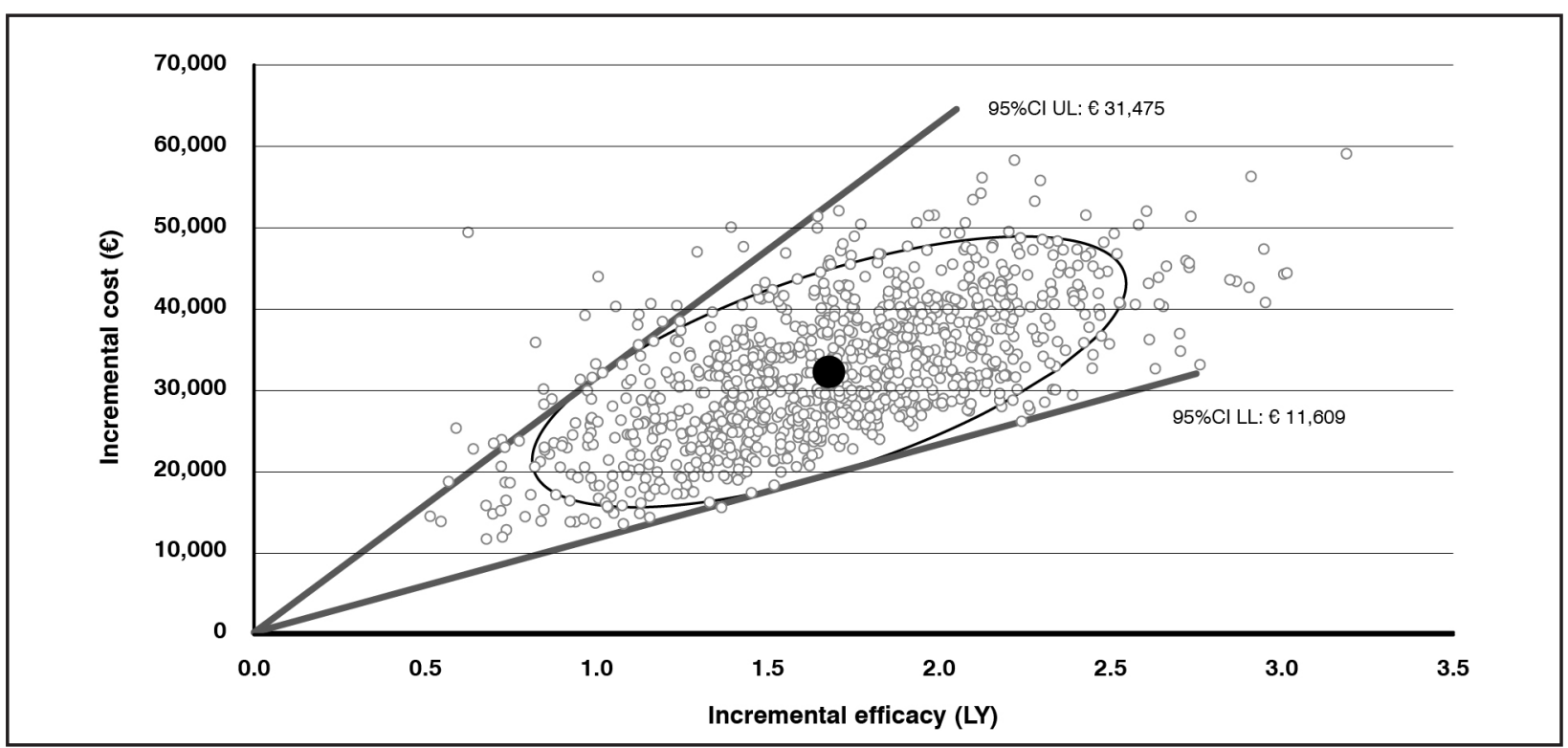

Figure 2. Probabilistic sensitivity analysis results

$\mathrm{Cl}=$ confidence interval; $\mathrm{LL}=$ lower limit; $\mathrm{LY}=$ life years; $\mathrm{UL}=$ upper limit 


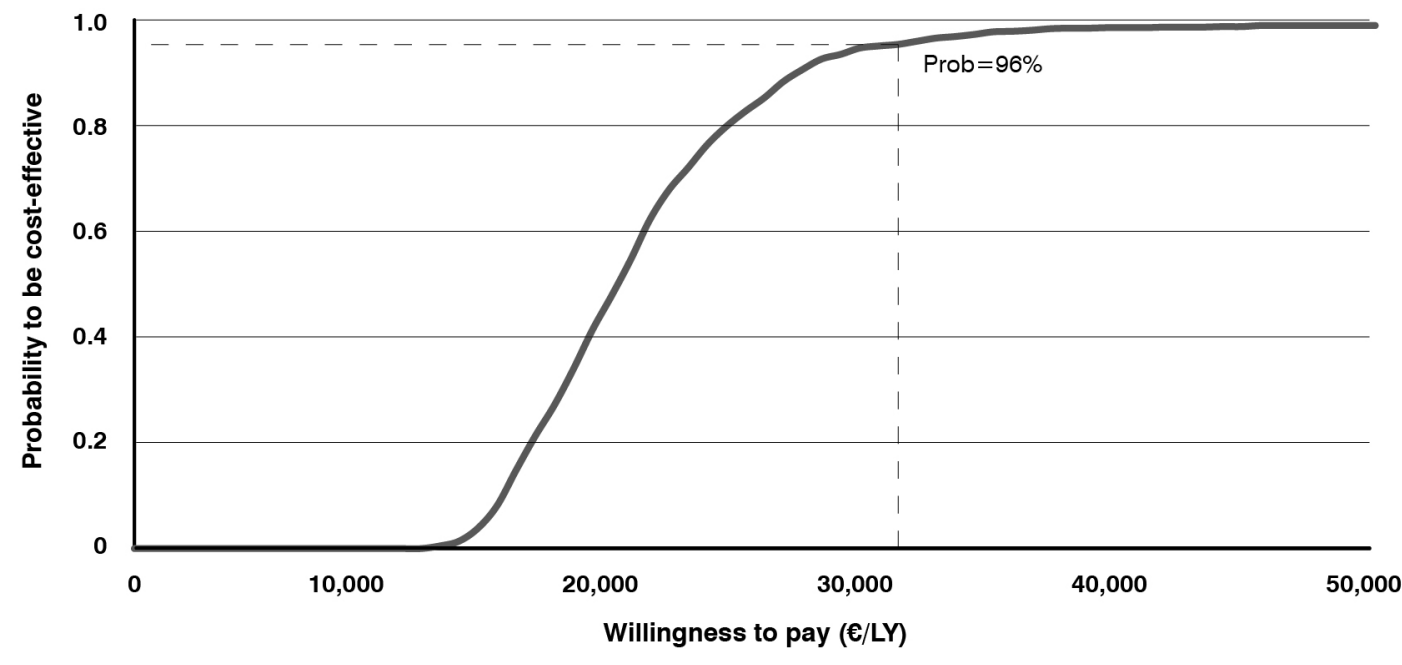

Figure 3. Cost-effectiveness acceptability curve

$L Y=$ life years

\section{DISCUSSION}

This cost-effectiveness analysis aimed to evaluate clinical and economic consequences of alirocumab, modeling the evolution of a cohort of hypothetical patients with or without the adjunction of alirocumab added to background therapy (maximum tolerated dose statins plus ezetimibe). The target population consists of patients with high baseline risk of CV events with previous hospitalization. Data were obtained from both Italian real world evidence regarding statins consumption [8] and UK-THIN, a large and well-validated electronic UK primary care database [9].

The analysis shows that alirocumab added to background therapy is more costly ( $€ 45,358$ vs $€ 13,208$ for MDTS + E) but more effective (8.01 LY vs 6.33 LY for MDTS+E) leading to an ICER of $€ 19,158$ per LY. Results of sensitivity analysis confirm base case analysis (95\% CI ICER: $€ 11,609-31,475)$; at a willingness to pay threshold of $€ 30,000$ per LY, alirocumab has an almost $96 \%$ probability to be cost effective vs. MDTS+E alone.

Even if data regarding risk of events do not derive from a national database, information used are comparable based on data found in the CORE report [21]. This document evaluates the burden of disease and healthcare costs related to about 6,000 patients treated with statins after a previous episode of acute coronary syndrome in Italy. Annual risk for CV events obtained from the CORE report $(26 \%)$ is comparable with data applied in our model (16\% for patients without diabetes and $25 \%$ for patients with diabetes) such as the mean age (COREreport mean age is 71 years; presented analysis: UK-THIN ACS subgroup mean age is 69.570.9 years).

Our results indicate that alirocumab in addition to MDTS $+\mathrm{E}$ is cost-effective compared to $\mathrm{MDTS}+\mathrm{E}$ alone in a representative cohort of high $\mathrm{CV}$ risk patients in Italy. Other economic analyses conducted in Norway [22] and the US [23] suggested that PCSK9 inhibitors are not cost-effective, in contrast with the presented model. Discrepancies could be related to differences between considered populations, different therapeutic schemes, and different economic agreements in involved countries. Populations could not be considered fully comparable; in Kazi et al. [23] for example, patients are younger (mean age 67.3 vs 74.7) and have more fa-

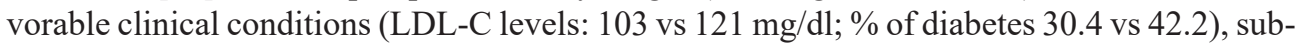
sequently, benefits estimated for the US population are smaller compared to patients considered in our model for which the effect of alirocumab is more evident. Moreover, comparisons between therapeutic schemes are different, Kazi and colleagues evaluate effects of alirocumab or ezetimibe added to statin therapy while our analysis considers statins plus ezetimibe with or without alirocumab.

Although the analysis of Korman et al. [22] highlights the improvement of effectiveness of PCSK9 inhibitors, authors find that these therapies would not be cost-effective. Differences between Norwegian and Italian data could be related to higher costs estimated and different therapeutic schemes evaluated. 


\section{Limitations}

Our study has some limitations. The model does allow transitions from the post-IS health states to the ACS event. This could be problematic in theory as the post-stroke health states are usually associated with lower utilities. Also, transition from the post-IS and post-ACS health states to the revascularization event are not admitted. Such assumptions were investigated in sensitivity pathway analysis (data not shown) and had a small impact on the ICER. Finally, national sources for CV baseline risk were not available and data from UK-THIN were used. However, potential differences among Italian and UK patients were partially reduced adjusting for age and LDL-C levels.

In conclusion, our analysis indicates that alirocumab in addition to MDTS+E is cost-effective versus MDTS+E alone for high CV risk patients in Italy. Specifically, the effectiveness increases in the patient subset with recent $\mathrm{CV}$ event $(<12$ months from index) indicating the potential benefit of alirocumab if started directly after an acute event, as add-on to background therapy.

\section{Funding}

This study and the article were funded by Sanofi Italy. Sanofi reviewed and provided feedback on the paper to the authors. The authors had full editorial control of the paper, and provided their final approval of all content.

\section{Conflicts of interest}

LP is co-owner and employee of AdRes, which has received project funding from Sanofi ltaly. MP is employee of AdRes.

AS, FF, and LG are employees of Sanofi.

\section{REFERENCES}

1. Yusuf S, Hawken S, Ounpuu S, et al. Effect of potentially modifiable risk factors associated with myocardial infarction in 52 countries (the INTERHEARTstudy): case-control study. Lancet. 2004; 364: 937-52; https://doi. org/10.1016/S0140-6736(04)17018-9

2. Ference BA, Ginsberg HN, Graham I, et al. Low-density lipoproteins cause atherosclerotic cardiovascular disease. 1. Evidence from genetic, epidemiologic, and clinical studies. A consensus statement from the European Atherosclerosis Society Consensus Panel. Eur Heart J. 2017; 38: 2459-72; https://doi.org/10.1093/eurheartj/ehx144

3. Goldstein JL, Brown MS, et al. A Century of Cholesterol and Coronaries: From Plaques to Genes to Statins. Cell. 2015; 161: 161-72; https://doi.org/10.1016/j.cell.2015.01.036

4. European Medicines Agency. Praluent ${ }^{\circledR}$ - Summary of product characteristics. Available at https://www.ema.europa. eu/documents/product-information/praluent-epar-product-information_en.pdf (last accessed May 2021)

5. Schwartz GG, Steg PG, Szarek M, et al; ODYSSEY OUTCOMES Committees and Investigators. Alirocumab and cardiovascular outcomes after acute coronary syndrome. $N$ Engl J Med. 2018; 379: 2097-107; https://doi. org/10.1056/NEJMoa1801174

6. Lakdawalla DN, Doshi JA, Garrison LP, et al. Defining Elements of Value in Health Care - A Health Economics Approach: An ISPOR Special Task Force Report [3]. Value in Health. 2018; 21: 131-9; https://doi.org/10.1016/j. jval.2017.12.007

7. Iannazzo S, Pradelli L, Carsi M, et al. Cost-effectiveness analysis of LHRH agonists in the treatment of metastatic prostate cancer in Italy. Value Health. 2011; 14: 80-9; https://doi.org/10.1016/j.jval.2010.10.023

8. Arca M, Ansell D, Averna M, et al. Statin utilization and lipid attainment in high or very-high cardiovascular risk patients: Insights from Italian general practice. Atherosclerosis. 2018; 271: 120-7; https://doi.org/10.1016/j.atherosclerosis.2018.02.024

9. National Institute for Health and Care Excellence (NICE). Alirocumab for treating primary hypercholesterolaemia and mixed dyslipidaemia. Technology appraisal guidance, 22 June 2016. Available at https://www.nice.org.uk/ guidance/ta393 (last accessed May 2021)

10. Cholesterol Treatment Trialists' (CTT) Collaboration, Baigent C, Blackwell L, Emberson J, et al. The effects of lowering LDL cholesterol with statin therapy in people at low risk of vascular disease: meta-analysis of individual data from 27 randomised trials. Lancet. 2012; 380: 581-90; https://doi.org/10.1016/S0140-6736(12)60367-5 
11. Wilson PW, D'Agostino R Sr, Bhatt DL, et al. An international model to predict recurrent cardiovascular disease. Am J Med. 2012; 125: 695-703; https://doi.org/10.1016/j. amjmed.2012.01.014

12. Smolina K, Wright L, Rayner M, et al. Long-Term Survival and Recurrence After Acute Myocardial Infarction in England, 2004 to 2010. Circ Cardiovasc Qual Outcomes. 2012; 5: 532-40; https://doi.org/10.1161/CIRCOUTCOMES.111.964700

13. Informatore Farmaceutico Online. Available at www.codifa.it (last access December 2018)

14. AIFA. Liste di trasparenza farmaci equivalenti. https://www.aifa.gov.it/liste-di-trasparenza (last accessed May 2021)

15. Ray JA, Valentine WJ, Secnik K, et al. Review of the cost of diabetes complications in Australia, Canada, France, Germany, Italy and Spain. Curr Med Res Opin. 2005; 21: 161729; https://doi.org/10.1185/030079905X65349

16. Tarricone R, Marchetti M, Lamotte M, et al. What reimbursement for coronary revascularization with drug-eluting stents? Eur J Health Econ. 2004; 49: 309-16; https://doi. org/10.1007/s10198-004-0258-x

17. HICPs - Harmonized Indices of Consumer Prices - Health sector. Available at: https:// ec.europa.eu/eurostat/databrowser/view/teicp060/default/table?lang=en (last accessed May 2021)

18. GU Serie Generale n.23 del 28-01-2013 - Suppl. Ordinario n. 8. Decreto 18 ottobre 2012. Remunerazione prestazioni di assistenza ospedaliera per acuti, assistenza ospedaliera di riabilitazione e di lungodegenza post acuzie e di assistenza specialistica ambulatoriale. (13A00528). Available at: https://www.gazzettaufficiale.it/eli/id/2013/01/28/13A00528/ sg (last accessed May 2021)

19. Wan X, Wang W, Liu J, Tong T. Estimating the sample mean and standard deviation from the sample size, median, range and/or interquartile range. BMC Med Res Methodol. 2014; 14: 135; https://doi.org/10.1186/1471-2288-14-135

20. Raftery J. NICE: faster access to modern treatments? Analysis of guidance on health technologies. BMJ. 2001; 323: 1300-3; https://doi.org/10.1136/bmj.323.7324.1300

21. Lucioni C, Mazzi S, Rossi E, et al. Therapeutic Strategies and Health Costs of Patients Admitted for a Cardiovascular Event in Italy. GRHTA. 2016; 3(2): 80-91; https://doi. org/10.5301/GRHTA.5000221

22. Korman M, Torbjørn W et al. Modelling the cost-effectiveness of PCSK9 inhibitors vs. ezetimibe through LDL-C reductions in a Norwegian setting. Eur Heart $J$ Cardiovasc Pharmacother. 2018; 4: 15-22; https://doi.org/10.1093/ehjcvp/pvx010

23. Kazi DS, Penko J, Coxson PG, et al. Cost-Effectiveness of Alirocumab: A Just-in-Time Analysis Based on the ODYSSEY Outcomes Trial. Ann Intern Med. 2019; 170: 221-9; https://doi.org/10.7326/M18-1776 


\section{APPENDIX}

\begin{tabular}{lccc}
\hline & Alirocumab+MDTS+E & MDTS+E & Delta \\
\hline LY & 7.98 & 6.30 & 1.68 \\
Total cost $(\boldsymbol{\epsilon})$ & $45,292.94$ & $13,251.10$ & $32,041.84$ \\
Drug cost & $36,986.91$ & $2,795.96$ & $34,190.95$ \\
CV events & $8,293.10$ & $10,455.14$ & $-2,162.04$ \\
- ACS & $4,299.70$ & $6,091.46$ & $-1,791.77$ \\
• Revascularization & 738.15 & 804.42 & -66.27 \\
- Non-Fatal IS & $1,426.15$ & $1,580.22$ & -154.07 \\
- CV deaths & $1,829.10$ & $1,979.04$ & -149.93 \\
LDL-C detection & 12.93 & 0.00 & 12.93 \\
ICER $(\boldsymbol{\epsilon})$ & & & $19,070.72$ \\
\hline
\end{tabular}

Table IA. Cost-effectiveness analysis: results for ACS 0-12 sub-population

ACS = acute coronary syndrome; CV = cardiovascular; $\mathrm{E}=$ ezetimibe; ICER = incremental cost effectiveness ratio; IS = ischemic stroke; LDL-C = lowdensity lipoprotein cholesterol; MDTS = maximum dose tolerated statins

\begin{tabular}{lccc}
\hline & Alirocumab+MDTS+E & MDTS+E & Delta \\
\hline LY & 8.55 & 6.91 & 1.63 \\
Total cost $(\boldsymbol{\epsilon})$ & $46,812.16$ & $12,261.33$ & $34,550.84$ \\
Drug cost & $39,600.88$ & $3,067.09$ & $36,533.80$ \\
CV events & $7,198.35$ & $9,194.24$ & $-1,995.89$ \\
• ACS & $3,419.62$ & $5,093.11$ & $-1,673.50$ \\
• Revascularization & 495.69 & 550.66 & -54.97 \\
• Non-Fatal IS & $1,502.29$ & $1,623.33$ & -121.04 \\
• CV deaths & $1,780.75$ & $1,927.14$ & -146.39 \\
LDL-C detection & 12.93 & 0.00 & 12.93 \\
ICER (€) & & & $21,155.68$ \\
\hline
\end{tabular}

Table IIA. Cost-effectiveness analysis: results for ACS $>12$ sub-population

ACS = acute coronary syndrome; $\mathrm{CV}=$ cardiovascular; $\mathrm{E}=$ ezetimibe; ICER = incremental cost effectiveness ratio; IS = ischemic stroke; LDL-C = Iowdensity lipoprotein cholesterol; MDTS = maximum dose tolerated statins 